\title{
Subarrayed Antenna Array Synthesis Using Ternary Adjusting Method
}

\author{
Guolong He, ${ }^{1,2}$ Yafeng Zhan, ${ }^{1}$ Yukui Pei, ${ }^{1}$ and Bin $\mathrm{Wu}^{2}$ \\ ${ }^{1}$ Space Centre, Tsinghua University, Beijing 100084, China \\ ${ }^{2}$ Beijing Institute of Tracking and Telecommunication Technology, Beijing 100094, China \\ Correspondence should be addressed to Guolong He; qingchunruyu@qq.com
}

Received 10 August 2014; Accepted 26 October 2014; Published 15 December 2014

Academic Editor: Chung-Huan Li

Copyright (c) 2014 Guolong He et al. This is an open access article distributed under the Creative Commons Attribution License, which permits unrestricted use, distribution, and reproduction in any medium, provided the original work is properly cited.

\begin{abstract}
Ternary adjusting method is proposed and combined with particle swarm optimization (PSO) algorithm for subarrayed antenna array synthesis. Ternary variables are introduced to represent element adjustments between adjacent subarrays. Compared to previous methods, rounding-off operations are not required any longer, and the equation constraint of the fixed total element number is also removed, which effectively reduces the complexity of implementation while obtaining improved topology exploration capability simultaneously.
\end{abstract}

\section{Introduction}

Antenna array technique plays a very important role in modern antenna, radar, and wireless communication systems. Many optimization techniques have been developed for antenna array synthesis to satisfy various requirements $[1-$ 8]. It is a well-known technique to partition large antenna arrays into contiguous subarrays in order to reduce cost via common use of components, mass production of identical subarrays, and simplified feed networks. Unfortunately, placing amplitude weights at the subarray ports will create grating lobes (GLs) due to the periodicity and larger spacing between subarrays. To address this problem, different techniques that aimed at breaking the periodicity have been proposed [9-13]. Recently, a method allowing each subarray to have a different number of elements was considered in $[12,13]$, where the subarray sizes and the amplitude weights are optimized jointly by genetic algorithm (GA) to reduce the sidelobe level (SLL) of the antenna array.

Since there are two different types of parameters in this optimization problem, that is, integers for the subarray sizes and real values for the amplitude weights, a hybrid variable vector is required to represent each candidate design and the corresponding process is the so-called mixed-integer optimization. Particle swam optimization (PSO), a recently developed evolutionary optimization algorithm by imitating behaviors of swarm during their food-searching activities, has been found to be very effective and robust and is an appealing technique for antenna array synthesis [14-24]. However, it is generally believed that GA is superior to PSO for discrete and mixed-integer optimization problems, because PSO was intended to deal with continuous optimization problems only while GA can translate a binary string to integer value directly. In previous works [19-23], evolutions of integer variables in PSO were often implemented by rounding-off associated real values that appear in the optimization process; thus, the performance is significantly deteriorated and some deficiencies have been reported [24]. Particles would easily be trapped in a local best solution because of the rounding-off process, which prevents them from reaching better solutions. Meanwhile, it is worth noticing that, due to the fixed total number of array elements, the updated subarray sizes must be modified to satisfy this constraint firstly during the optimization process.

This paper proposes a novel method, named ternary adjusting method, for subarray size updating, which does not require rounding-off operations any longer while removing the constraint of the fixed total element number simultaneously. The rest of this paper is organized as follows. First in Section 2, ternary variables are introduced to represent 


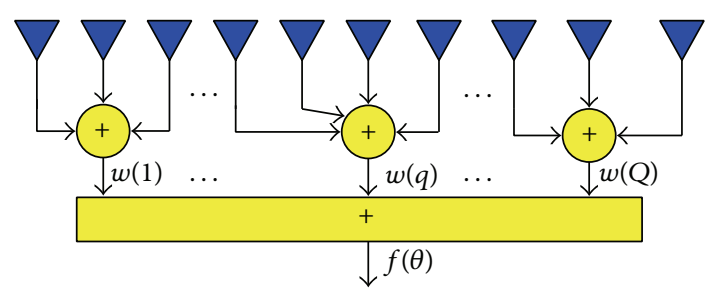

FIgURE 1: Geometry of the subarrayed antenna array.

element adjustments between adjacent subarrays, and the novel subarray size optimization method is presented. Then in Section 3, two previous examples are revisited and better results are obtained. Lastly, this paper is summarized in Section 4 .

\section{Ternary Adjusting Method}

As shown in Figure 1, an $\mathrm{N}$-element linear antenna array is equally spaced along the $x$-axis and partitioned into $Q$ subarrays, with unequal sizes and different amplitude weights at the subarray output ports. The corresponding array factor $f(\theta)$ is given by

$$
f(\theta)=\sum_{q=1}^{Q} w(q) \sum_{i=1}^{n(q)} \cos \left(2 \pi x_{n} \sin \theta\right)
$$

where $x_{n}$ is the position of the $n$th element in wavelength, $\theta$ is the angle relative to boresight, and $n(q)$ and $w(q)$ are number of elements and the amplitude weight of the qth subarray, respectively. During the iterations, the amplitude weight vector $\mathbf{w}$ is updated with the velocity vector $\mathbf{v}$ following the classical PSO scheme

$$
\begin{aligned}
\mathbf{v}_{k+1}= & \alpha \times \mathbf{v}_{k}+c_{1} \times \gamma_{1} \times\left(\mathbf{w}_{\text {pbest }, k}-\mathbf{w}_{k}\right) \\
& +c_{2} \times \gamma_{2} \times\left(\mathbf{w}_{\text {gbest }, k}-\mathbf{w}_{k}\right), \\
\mathbf{w}_{k+1}= & \mathbf{w}_{k}+\mathbf{v}_{k},
\end{aligned}
$$

where $\alpha$ is the inertial weight, $c_{1}$ and $c_{2}$ are acceleration constants that specify how much each particle is influenced by the personal best solution, pbest, and the global best solution ever found by the swarm, gbest, and $\gamma_{1}$ and $\gamma_{2}$ are two random variables uniformly distributed on $[0,1]$.

Since the array has a fixed total number of array elements, it is coming into mind that the subarray size updating process can be regarded as element adjustments between adjacent subarrays. In other words, the antenna array is divided into $Q$ subarrays by $Q-1$ dividers. Therefore, as shown in Figure 2, a random vector $\mathbf{m}$ consisting of $Q-1$ ternary variables

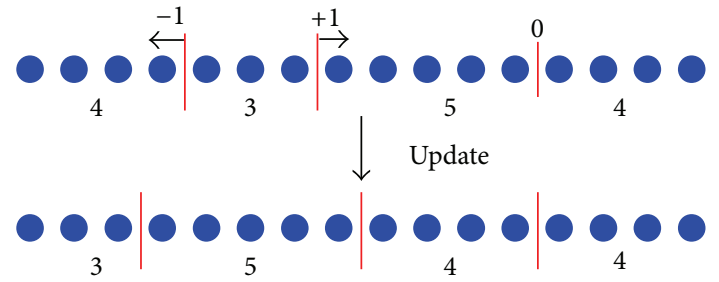

FIGURE 2: An example of ternary adjusting method with $N=16$ and $Q=4$, the integer vector is updated from $[4,3,5,4]$ to $[3,5,4,4]$ with $\mathbf{m}=[-1,1,0]$.

is introduced, and the subarray size vector $\mathbf{n}$ is updated as follows:

$$
n(q)= \begin{cases}n_{\text {gbest }, k}(q)+m_{k}(q+1), & \text { for } q=1, \\ n_{\text {gbest }, k}(q)-m_{k}(q)+m_{k}(q+1), & \text { otherwise, } \\ n_{\text {gbest }, k}(q)-m_{k}(q), & \text { for } q=Q,\end{cases}
$$

where $m(q)=$ rand $\{-1,0,+1\}$,

where $m(q)=0$ represents no adjustment between the $q$ th and $q+1$ th subarrays, +1 represents moving one element from the $q+1$ th subarray to the $q$ th subarray, and -1 represents the opposite operation, respectively. In (3), because the values of the updated subarray sizes are always integers, no roundingoff operations are needed any longer. In addition, the adjusted elements are just moved from one subarray to another; thus, the number of the total elements keeps unchanged and this constraint is also removed, which effectively reduces the complexity of implementation.

Here for a given subarray, each time only the elements at the ends could be adjusted. Therefore, the updated subarray size vector is restricted nearby the former one, which is a reasonable area to search for better solutions. The motivation is supported by nature. Too large adjustments would make the updated subarray sizes not matched with the present amplitude weights, which will cause performance degradation rather than improvements. Moreover, the random ternary vector makes the updating and searching operations continued in the whole duration of the optimization process, which can prevent particles from being trapped in local best solutions and lead to improved topology exploration capability, especially at the later stage of the optimization process.

\section{Examples and Results}

3.1. Sidelobe Reduction for the Linear Array. The first example is to reduce SLLs/GLs of a 128-element linear array, which has been investigated by GA in [12]. The array is partitioned into 16 subarrays, and the distance between the elements is $0.5 \lambda$. The number of elements in each subarray is restricted to be even for engineering practice consideration; thus, each time one pair of elements are adjusted as a whole between adjacent subarrays. The parameters of PSO algorithm are 


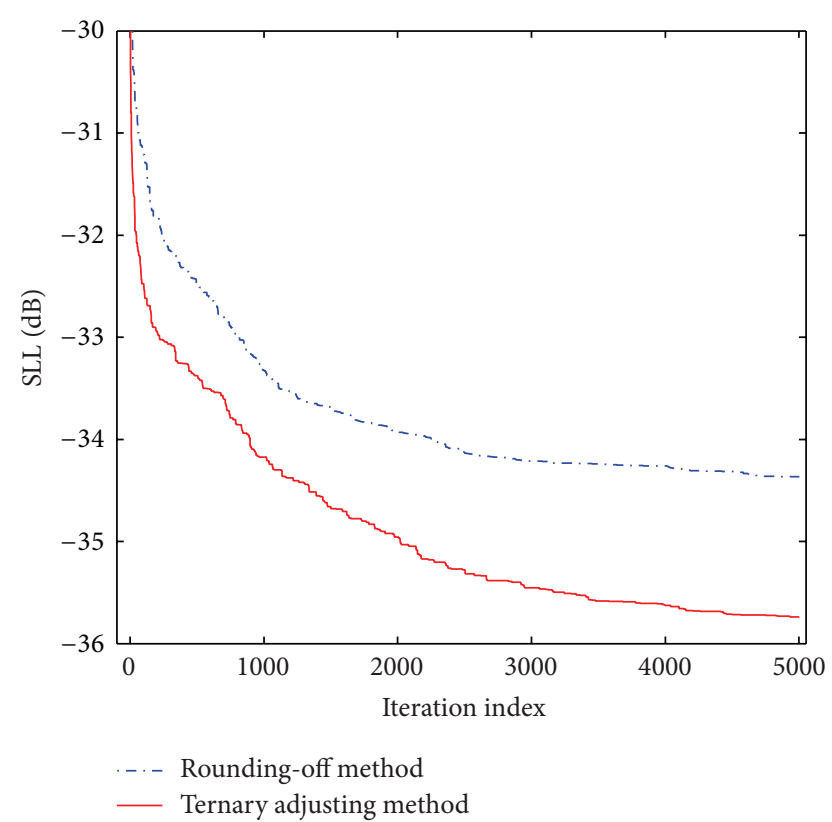

Figure 3: Comparison of global best values averaged over 20 independent trials.

set as suggested by previous literatures [14-18, 24], and $\alpha$ is decreased linearly from 0.9 to 0.4 during iterations, and $c_{1}=c_{2}=2$ for better convergence performance. A 20 agent swarm is used in optimization for 5000 iterations.

Figure 3 illustrates the convergence of global best values averaged over 20 independent trials. An averaged global best value of $-35.74 \mathrm{~dB}$ is obtained by ternary adjusting method, compared with $-34.36 \mathrm{~dB}$ by conventional rounding-off method. During the 20 trails, the best solution ever found by ternary adjusting method is $-36.5 \mathrm{~dB}$, which is all better than uniform tapering $(-13.3 \mathrm{~dB})$, rounding-off method $(-35.7 \mathrm{~dB})$, and GA $(-35.9 \mathrm{~dB})$. These results have clearly showed that ternary adjusting method has better convergence performance and improved topology exploration capability than existing methods. Figure 4(a) illustrates the optimized subarray sizes and amplitude weights, where $\mathbf{n}=[14,8,6,8,6,4,8,10]$ and $\mathbf{w}=[1.0000,0.8491,0.7175$, $0.5310,0.4160,0.3173,0.2021,0.1048]$, respectively. Since the array is symmetric, only the right hand side is shown. The corresponding optimized array pattern is illustrated in Figure 4(b).

3.2. Sidelobe Reduction for the Planar Array. The ternary adjusting method described in this paper can be further expanded to planar array easily. The second example is about sidelobe reduction of a planar phased array with $24 \times 32$ elements investigated in [13]. The distance between each element is $0.5 \lambda$, and the scan range is $-45^{\circ} \leq \phi \leq 45^{\circ}$, $-10^{\circ} \leq \theta \leq 10^{\circ}$. In the $x$-direction the array is divided into 12 subarrays; thus, each subarray has two elements equally but with different amplitude weights, while in the $y$-direction the array is divided into 8 subarrays with unequal sizes.

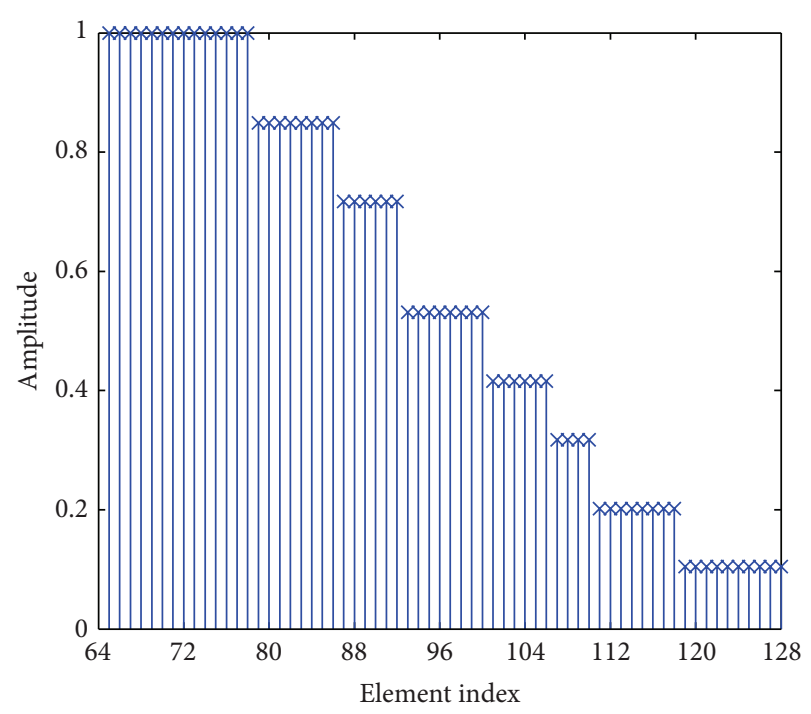

(a)

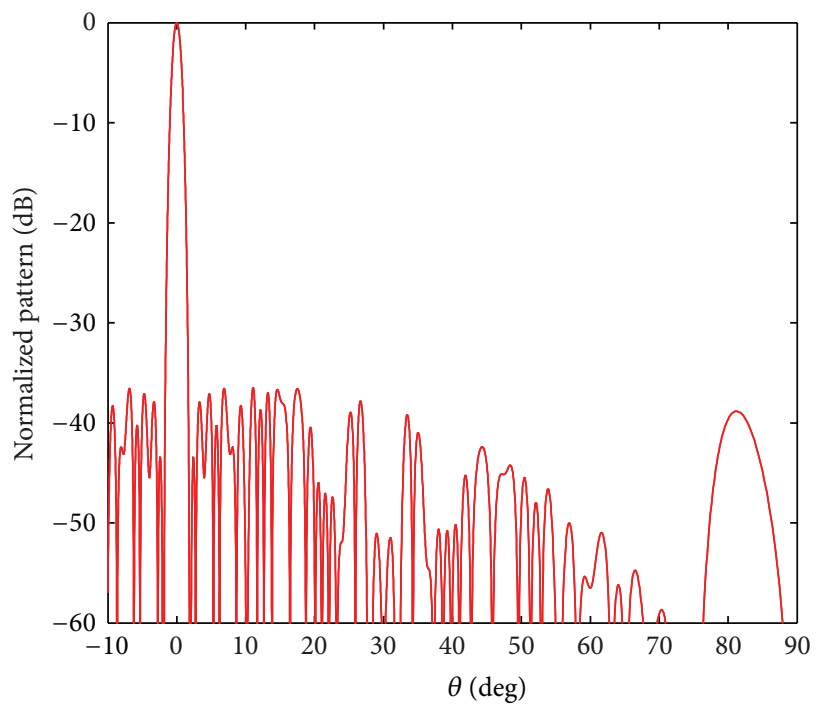

(b)

FIgURE 4: The optimization results of the linear array: (a) the optimized subarray sizes and amplitude weights and (b) the optimized array pattern.

Again, number of elements in each subarray is restricted to be even, symmetries are assumed in both directions, and parameters of PSO algorithm are set the same as those in the first example. A 20 agent swarm is used in optimization for 1000 iterations. The optimized configuration of the planar array is shown in Figure 5(a) with $\mathbf{n}_{\mathbf{y}}=[2,6,4,4]$, $\mathbf{w}_{\mathbf{x}}=[1.0000,0.8791,0.7514,0.6478,0.4065,0.3461]$, and $\mathbf{w}_{\mathbf{y}}=[1.0000,0.7485,0.5084,0.2143]$. Figure 5(b) illustrates the corresponding $3 \mathrm{D}$ array pattern in the worst case steered to $\left(-45^{\circ}, 10^{\circ}\right)$. The peak SLL of the optimized design is $-25.9 \mathrm{~dB}$, and more than $5 \mathrm{~dB}$ improvement is obtained compared to the previous result $(-20.7 \mathrm{~dB})$ in [13]. 


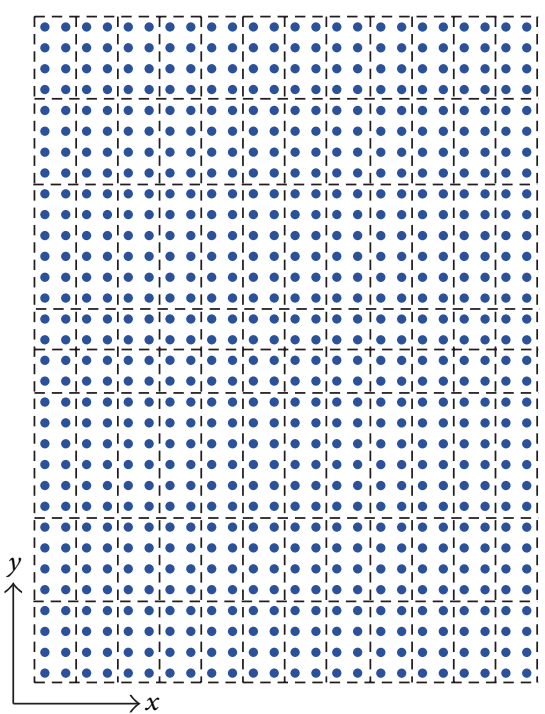

(a)

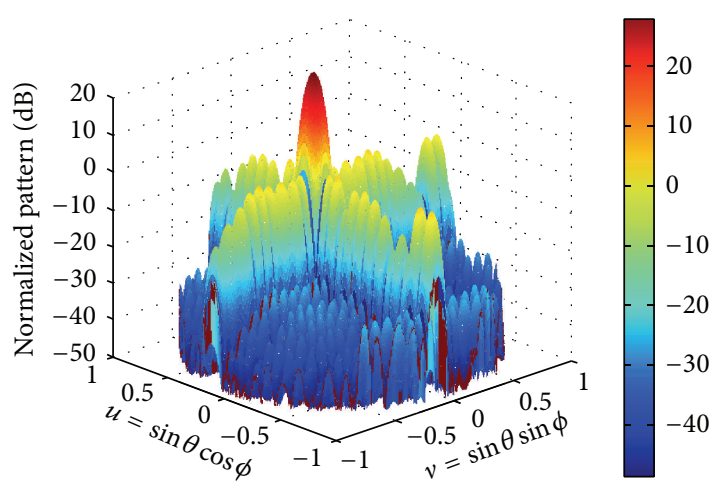

(b)

FIGURE 5: The optimization results of the planar array: (a) the optimized configuration and (b) the optimized 3D array pattern steered to $\left(-45^{\circ}, 10^{\circ}\right)$.

\section{Conclusion}

Synthesis of subarrayed antenna arrays involves mixedinteger optimization, that is, integers for the subarray sizes and real values for the amplitude weights. This paper has proposed a novel method, named ternary adjusting method, for subarray size updating, which does not require roundingoff operations any longer while removing the constraint of the fixed total element number simultaneously. This novel method was further combined with PSO algorithm and then applied to two examples about subarrayed antenna array synthesis. Numerical results have showed that ternary adjusting method has better convergence performance and improved topology exploration capability than existing methods. For such fixed length dividing problems, this novel method not only can be combined with PSO algorithm but also will be very useful for other evolutionary algorithms.

\section{Conflict of Interests}

The authors declare that there is no conflict of interests regarding the publication of this paper.

\section{Acknowledgments}

This work was supported by the National Natural Science Foundation of China (61271265, 61032003, and 61132002), National High Technology Research and Development Program (2012AA121605), and Tsinghua University Initiative Scientific Research Program (2011Z05112).

\section{References}

[1] R. S. Elliott, Antenna Theory and Design, Wiley-Interscience, IEEE, Hoboken, NJ, USA, 2003.

[2] R. J. Mailloux, Phased Array Antenna Handbook, Artech House, Boston, Mass, USA, 2005.

[3] R. L. Haupt, Antenna Arrays: A Computational Approach, John Wiley \& Sons, New York, NY, USA, 2010.

[4] M. M. Khodier and C. G. Christodoulou, "Linear array geometry synthesis with minimum sidelobe level and null control using particle swarm optimization," IEEE Transactions on Antennas and Propagation, vol. 53, no. 8, pp. 2674-2679, 2005.

[5] D. W. Boeringer and D. H. Werner, "Particle swarm optimization versus genetic algorithms for phased array synthesis," IEEE Transactions on Antennas and Propagation, vol. 52, no. 3, pp. 771-779, 2004.

[6] M. A. Panduro, D. H. Covarrubias, C. A. Brizuela, and F. R. Marante, "A multi-objective approach in the linear antenna array design," International Journal of Electronics and Communications AEUE, vol. 59, no. 4, pp. 205-212, 2005.

[7] W. T. Li, X. W. Shi, and Y. Q. Hei, "An improved particle swarm optimization algorithm for pattern synthesis of phased arrays," Progress in Electromagnetics Research, vol. 82, pp. 319-332, 2008.

[8] G. He and B. Wu, "Unified particle swarm optimization with random ternary variables and its application to antenna array synthesis," Journal of Electromagnetic Waves and Applications, vol. 28, no. 6, pp. 752-764, 2014.

[9] D. Petrolati, P. Angeletti, and G. Toso, "A lossless beam-forming network for linear arrays based on overlapped sub-arrays," IEEE Transactions on Antennas and Propagation, vol. 62, no. 4, pp. 1769-1778, 2014.

[10] N. Toyama, "Aperiodic array consisting of subarrays for use in small mobile earth stations," IEEE Transactions on Antennas and Propagation, vol. 53, no. 6, pp. 2004-2010, 2005.

[11] P. Rocca, R. L. Haupt, and A. Massa, "Sidelobe reduction through element phase control in uniform subarrayed array antennas," IEEE Antennas and Wireless Propagation Letters, vol. 8, pp. 437-440, 2009.

[12] R. L. Haupt, "Optimized weighting of uniform subarrays of unequal sizes," IEEE Transactions on Antennas and Propagation, vol. 55, no. 4, pp. 1207-1210, 2007.

[13] H. Wang, D.-G. Fang, and Y. L. Chow, "Grating lobe reduction in a phased array of limited scanning," IEEE Transactions on Antennas and Propagation, vol. 56, no. 6, pp. 1581-1586, 2008.

[14] J. Kennedy and R. Eberhart, "Particle swarm optimization," in Proceedings of the IEEE International Conference on Neural Networks, vol. 4, pp. 1942-1948, December 1995. 
[15] R. Poli, "Analysis of the publications on the applications of particle swarm optimisation," Journal of Artificial Evolution and Applications, vol. 2008, Article ID 685175, 10 pages, 2008.

[16] J. Robinson and Y. Rahmat-Samii, "Particle swarm optimization in electromagnetics," IEEE Transactions on Antennas and Propagation, vol. 52, no. 2, pp. 397-407, 2004.

[17] N. Jin and Y. Rahmat-Samii, "Advances in particle swarm optimization for antenna designs: real-number, binary, singleobjective and multiobjective implementations," IEEE Transactions on Antennas and Propagation, vol. 55, no. 3, pp. 556-567, 2007.

[18] Y. Rahmat-Samii, J. M. Kovitz, and H. Rajagopalan, "Natureinspired optimization techniques in communication antenna designs," Proceedings of the IEEE, vol. 100, no. 7, pp. 2132-2144, 2012.

[19] A. Modiri and K. Kiasaleh, "Modification of real-number and binary PSO algorithms for accelerated convergence," IEEE Transactions on Antennas and Propagation, vol. 59, no. 1, pp. 214-224, 2011.

[20] E. C. Laskari, K. E. Parsopoulos, and M. N. Vrahatis, "Particle swarm optimization for integer programming," in Proceedings of the IEEE Congress on Evolutionary Computation (CEC '02), vol. 2, pp. 1582-1587, usa, May 2002.

[21] Z. Gaing, "Constrained optimal power flow by mixed-integer particle swarm optimization," in Proceedings of the IEEE Power Engineering Society General Meeting, vol. 1, pp. 243-250, June 2005.

[22] D. Liu, Q.-Y. Feng, and W.-B. Wang, "Discrete optimization problems of linear array synthesis by using real number particle swarm optimization," Progress in Electromagnetics Research, vol. 133, pp. 407-424, 2013.

[23] A. Hoorfar, J. Zhu, and S. Nelatury, "Electromagnetic optimization using a mixed-parameter self-adaptive evolutionary algorithm," Microwave and Optical Technology Letters, vol. 39, no. 4, pp. 267-271, 2003.

[24] N. Jin and Y. Rahmat-Samii, "Hybrid real-binary particle swarm optimization (HPSO) in engineering electromagnetics," IEEE Transactions on Antennas and Propagation, vol. 58, no. 12, pp. 3786-3794, 2010. 

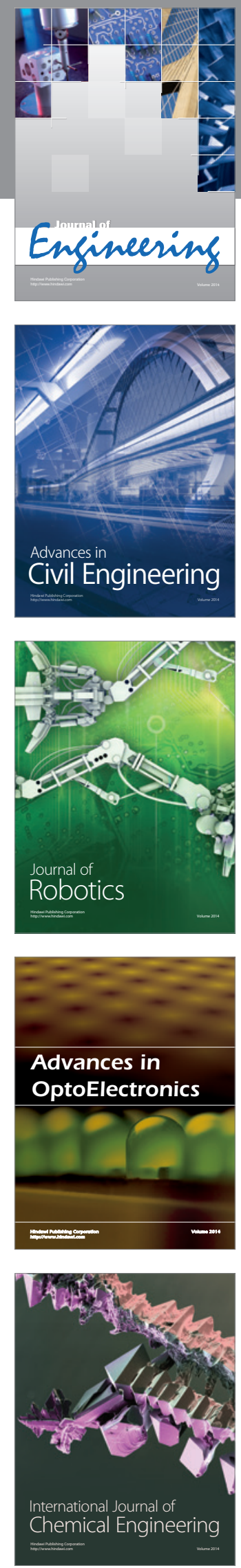

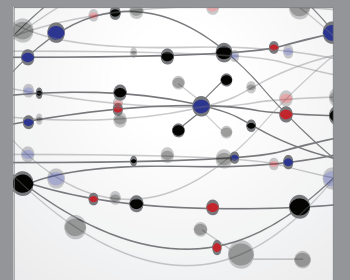

The Scientific World Journal
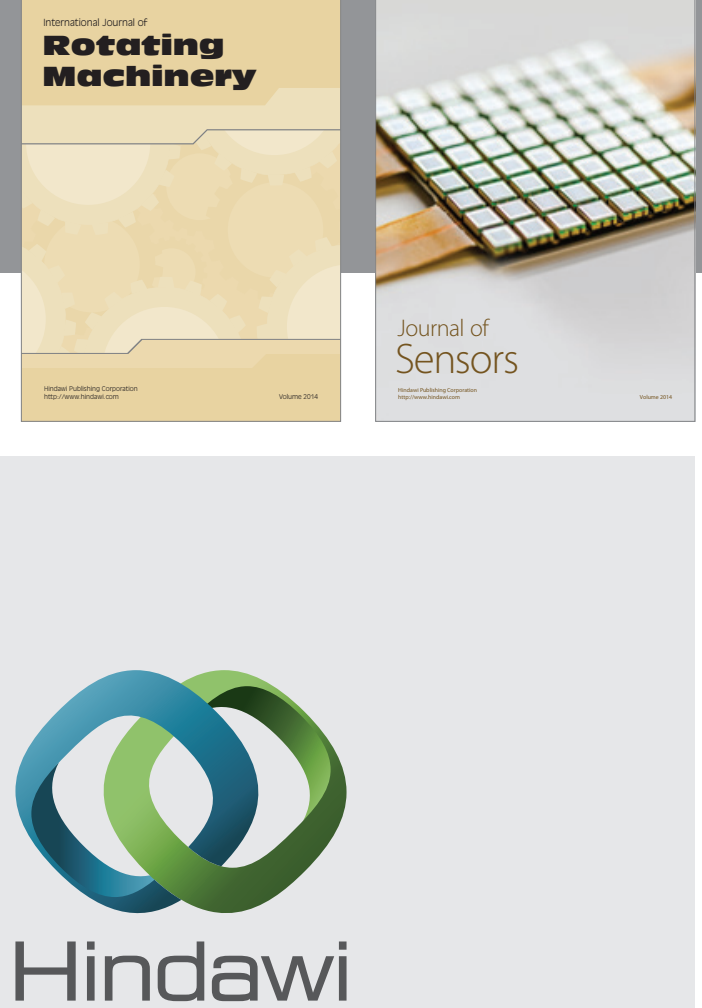

Submit your manuscripts at http://www.hindawi.com
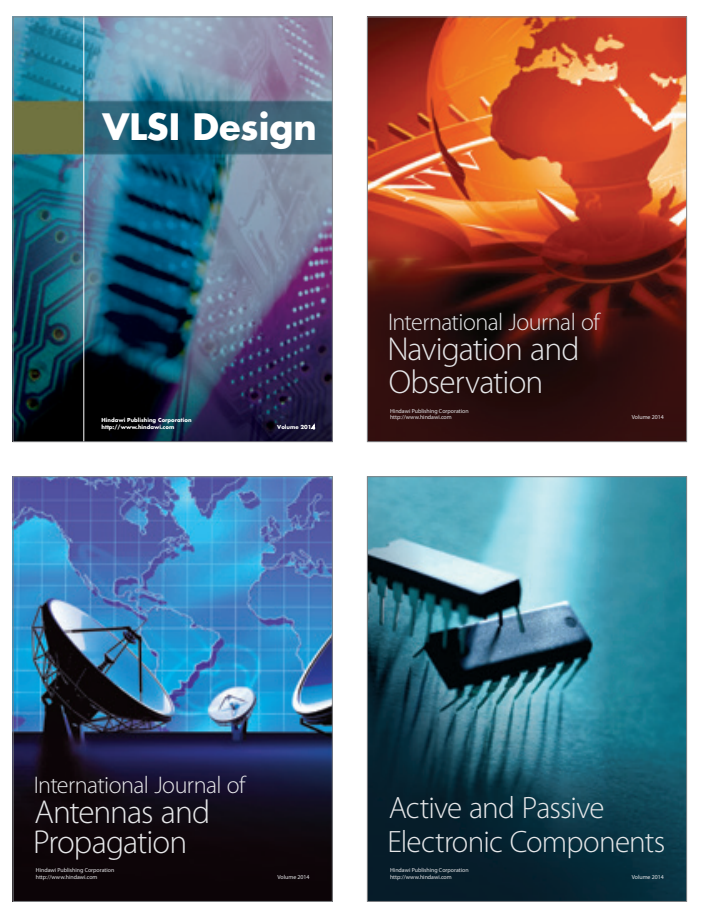
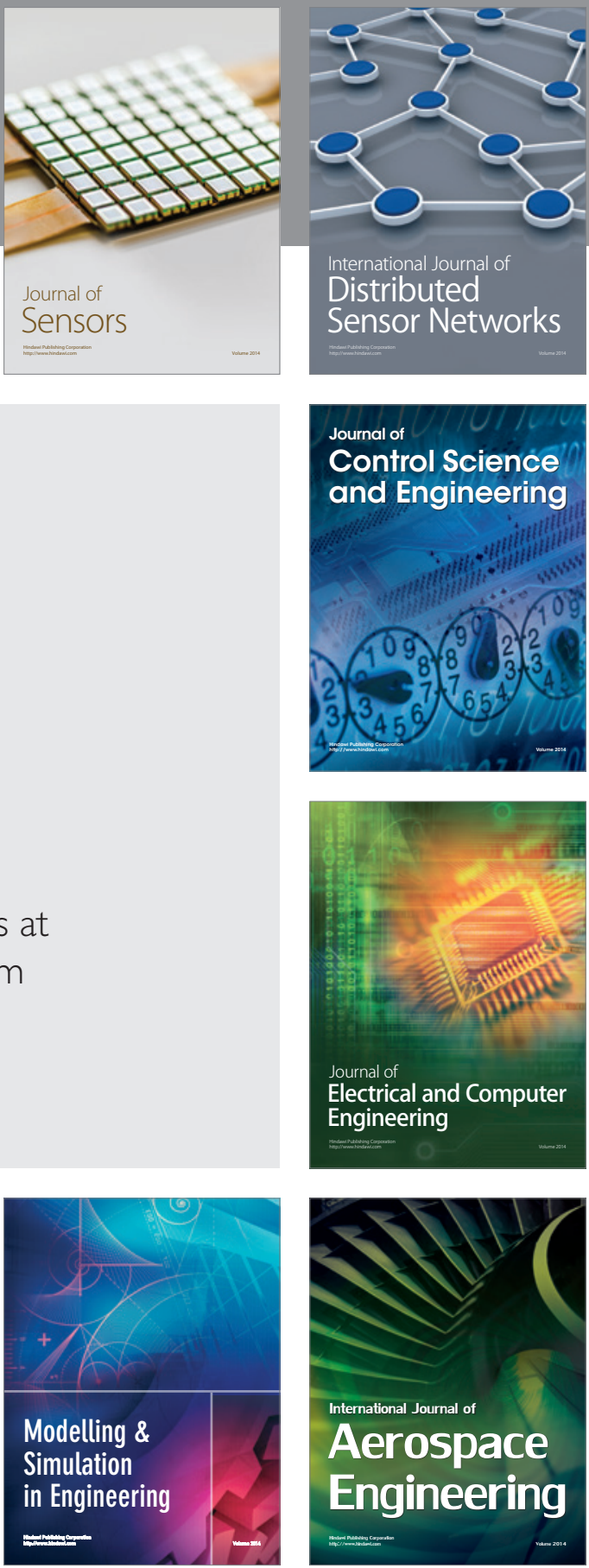

Journal of

Control Science

and Engineering
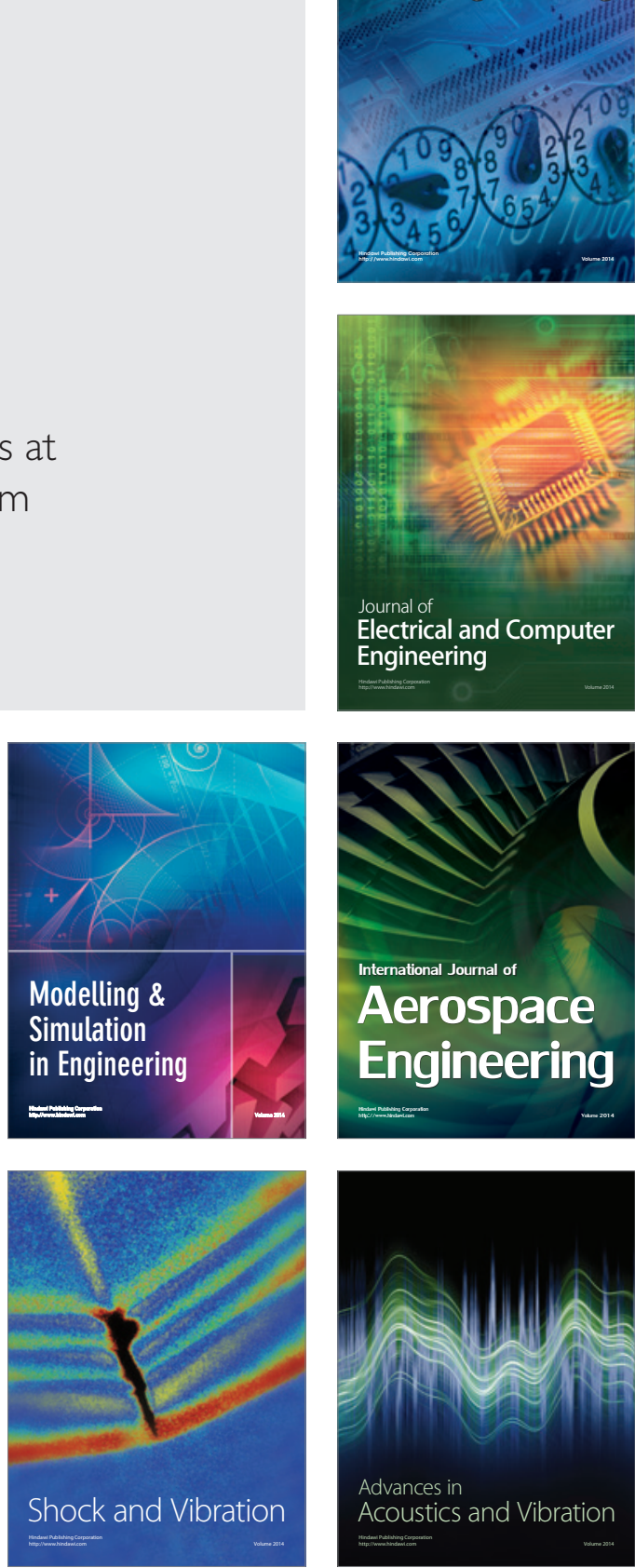